\title{
Stimulus configuration and line orientation in the horizontal-vertical illusion*
}

\author{
ELIZABETH O. CORMACK and ROBERT H. CORMACK† \\ New Mexico Institute of Mining and Technology, Socorro, New Mexico 87801
}

\begin{abstract}
The method of average error was used with a mixed design to measure the horizontal-vertical illusion (HVI) for 40 Ss. Six stimulus configurations $(L\rfloor,, \vdash,-, L,+)$ were combined with seven angular orientations of the upright standard, and on each trial the variable horizontal was adjusted to appear equal to the standard in length. Results showed that for no stimulus configuration did the vertical orientation of the standard yield the greatest illusion. The magnitude of the HVI was dependent upon the stimulus configuration, upon the orientation of the standard, and upon an interaction between these variables. For the $\perp$ and + , equal inclinations of the standard to either side of the vertical yielded equal effects; for the other figures, asymmetrical effects were produced. The results are discussed in relation to the perspective theory of visual illusions.
\end{abstract}

In the horizontal-vertical illusion (HVI), upright lines tilted slightly (15-30 deg) from the vertical result in a greater illusion relative to the horizontal than do vertical uprights (Morinaga, Noguchi, \& Ohishi, 1962; Pollock \& Chapanis, 1952; Shipley, Nann, \& Pentield, 1949; Underwood, 1966). Further, Pollock and Chapanis, and Underwood have shown that tilts of the upright to the left produce a slightly greater amplification of the illusion than do similar tilts to the right. Pollock and Chapanis presented their Ss with two unconnected lines located either side by side or one above the other on a vertical screen. Underwood presented Ss with a card containing a standard line drawn at some orientation, and asked them to draw a horizontal line of the same length.

A study by one of the authors (Cormack, 1969) observed the effects of the inclination of the vertical in a symmetrical figure $(+)$. The results suggested that while slight inclinations to either side of the vertical increased the illusion, the effects were equal for tilts in either direction. It seemed possible that an important factor in the differences between Cormack's data and those of other workers was the stimulus configuration employed.

A number of studies have shown that the spatial arrangement of the horizontal and vertical

\footnotetext{
*The authors are grateful to Kathy Branch and Chris Buys for their critical reading of this paper. Errors which remain are, of course, not their responsibility.

†Requests for reprints should be sent to Robert H. Cormack, Psychology Department, New Mexico Tech, Socorro, New Mexico 87801.
}

components, i.e., the stimulus configuration, is an important determinant of illusion magnitude. Künnapas (1955a), who studied the HVI extensively, showed that the inverted $\mathrm{T}(1)$ produced a greater illusion than the side $T(\vdash)$ and, in fact, found that the side $T$ gave a negative illusion, that is, the horizontal line appeared longer than the vertical. He also showed that the point at which the dividing line (vertical in the $\mathrm{T}$ and inverted $\mathrm{T}$ and horizontal in the side $T$ ) meets the divided line was an important variable. With the $T$ and inverted $T$, illusion magnitude increased as the dividing line approached the center of the divided line. With the side-T illusion, magnitude decreased as the dividing line approached the center. He inferred that these results indicated the effects of two illusions in his figures, an overestimation of the vertical line and an overestimation of the dividing line. He suggested that the latter effect was variable, depending upon the point of intersection, and that the two illusions summated in the $\mathrm{T}$ and inverted $\mathrm{T}$ but opposed one another in the side T. Tedford and Tudor (1969) and Finger and Spelt (1947) also compared the inverted and side-T figures. Both confirmed that the inverted $T$ yielded a greater illusion than the side $T$. In agreement with Künnapas, Tedford and Tudor found a reversal of the illusion with the side $T$ (i.e., a greater divided-line illusion), while Finger and Spelt found a small, but positive, illusion (a greater HVI).

In 1971, the present authors reported an attempt to study the effects of inclination of the vertical using four conventional HVI figures in which the horizontal and vertical components are connected (Cormack \& 
Cormack,.1971). They employed an $L$ figure ( $L)$, an inverted $T(1)$, a side $T(\vdash)$, and a cross $(+)$. They found that the effects of tilt were dependent on the figure used. The inverted $T$ and the cross gave results similar to those of Pollock and Chapanis (1952) and Underwood (1966) but with less, if any, right-left asymmetry. The $\mathrm{L}$ and side $\mathrm{T}$ gave much greater effects for tilts of the upright to the left than to the right. Notice that these latter effects occurred for stimulus figures asymmetric about the vertical axis.

If the right-left differences in the results obtained with the $L$ and side $T$ are due to their asymmetry, then mirror images of these figures should produce effects in the opposite direction. The present study was conducted to explore this possibility and, particularly, to examine further, under carefully controlled conditions, the effects of tilts of the upright on otherwise symmetrical figures (cross and inverted $\mathrm{T}$ ).

\section{METHOD}

\section{Apparatus}

The apparatus consisted of a plain black screen with a $2 \mathrm{~mm} \mathrm{x}$ $15 \mathrm{~cm}$ horizontal slot. Behind the slot were guides which held a $20 \times 100 \mathrm{~cm}$ black cardboard slide oriented vertically. On this slide was a white wedge-shaped section ranging in width from 5 to $15 \mathrm{~cm}$. The slide could be moved up and down within the guides, exposing a longer or shorter white line through the horizontal slot. This line served as the variable stimulus. Three such slides were prepared. On one, the white wedge had a vertical edge on the right, so that as it was moved the left end of the line lengthened and shortened. On another, the vertical edge was on the left, and on the third, the edges slanted symmetrically so that the exposed line changed equally on both ends. The backs of the slides were calibrated, allowing the length of the exposed white line to be read to the nearest millimeter.

It might be noted here that the use of a wedge-shaped section to change line length results in a tilt to one or both ends of the horizontal line. This tilt is about 6 deg from the vertical for the asymmetric wedges and $3 \mathrm{deg}$ for the symmetric wedge. The exposed segment, $2 \mathrm{~mm}$ at $160 \mathrm{~cm}$, subtended $4.3 \mathrm{~min}$ va (visual angle). While the tilt was not apparent in direct observation, it is not impossible that it may have had some slight effect on Ss' judgments.

On the front of the screen were guides into which could be placed $20 \times 25 \mathrm{~cm}$ clear plastic sheets. On each sheet was a standard $10 \mathrm{~cm} \times 2 \mathrm{~mm}$ white line positioned to intersect and form a specific angle with the adjustable horizontal line. Six stimulus configurations were used: $L$ figure $(L)$, side $T(-)$, inverted $T(L)$, cross $(+)$, reversed $L(\lrcorner)$, and reversed side $T(-1)$. For the $L$ and side $T$, the slide with a vertical edge on the left of the white wedge was placed behind the horizontal slot. For the inverted Tand cross, the slide with a vertical edge on the left of the white wedge was placed behind the horizontal slot. For the inverted $T$ and cross, the slide with the symmetrical wedge was used. The remaining slide was used with the reversed $L$ and reversed side $T$. Seven angular orientations of the standard line $(22.5,45,67.5,90,112.5,135$, and $157.5 \mathrm{deg}$ from the horizontal) were combined, with each configuration forming 42 distinct stimulus figures.

$S$ viewed the screen through a 5-mm circular viewing aperture located $160 \mathrm{~cm}$ from the screen and through a black reduction screen with an $8 \times 10 \mathrm{~cm}$ aperture located $95 \mathrm{~cm}$ from the black stimulus screen. The black screen carrying the standard and adjustable lines and the reduction screen were illuminated by incandescent lamps positioned to minimize brightness gradients, shadows, and highlights.

Although the reduction screen and the background against which the stimuli were presented were both black and care was taken to equalize illumination of the main screen and reduction screen, it proved impossible to prevent the edges of the aperture in the reduction screen from being faintly visible. The aperture subtended $8.8 \mathrm{deg}$ va horizontally and $7.1 \mathrm{deg}$ va vertically. The standard line subtended $3.6 \mathrm{deg}$ va, while the variable horizontal line could subtend from 1.8 to $5.4 \mathrm{deg}$ va. The stimulus figure was centered in the aperture, and since Künnapas $(1955 \mathrm{~b}, 1959)$ has shown that the size and shape of the visual field can affect the HVI, it is possible that the presence of these faint contours could have influenced illusion magnitude judgments. Data relevant to this possibility are mentioned in the discussion section.

\section{Subjects}

Nineteen females and 21 males, ranging in age from 18 to 43 years, served as Ss. Most were college students, all had at least $20 / 30$ vision, or vision corrected to $20 / 30$ with glasses or contact lenses.

\section{Procedure}

Ss were tested individually. They were seated at a table, behind a black screen within which was mounted the 5 -mm viewing aperture. They were instructed to use always the same eye for viewing throughout the experiment and to look away from the viewing aperture following each judgment. They were instructed to make the two lines the same length by directing $E$ to lengthen or shorten the horizontal line "a lot longer/shorter" (4 mm change), "longer/shorter" ( $2 \mathrm{~mm})$, or "a littie longer/shorter" $(1 \mathrm{~mm})$. They were allowed unlimited adjustments until satisfied that the lines were the same length. Two practice trials were given, and questions as to procedure were allowed. Each $S$ was then given 28 trials. A mixed design was employed. Because we were particularly interested in right-left asymmetry in symmetrical figures, all $40 \mathrm{Ss}$ observed these two figures (cross and inverted $T$ ). Twenty Ss (Group 1) observed the asymmetric figures with the standard on the left ( $L$ and side $T$ ), while the remaining 20 (Group 2) observed the same figures with the standard on the right (reversed $L$ and reversed side $\mathrm{T}$ ). Each $\mathrm{S}$ was presented all seven line orientations for each figure observed. The order of stimulus presentation was randomized across Ss. The adjustable horizontal line was set initially at or near the maximum or minimum line length, determined randomly.

It should be noted here that Gardner and Long (1960a, b) have shown that when the vertical is used as the standard, the obtained measures of the HVI tend to be larger than when the horizontal is used as the standard (an effect they call "errors of the standard"). Since, in the present study, the upright line was used as the standard, the constant errors in judgment (i.e., illusion magnitudes) may be inflated. However, this seemed preferable to the alternative of requiring $\mathrm{S} s$ to perform different tasks under the different stimulus conditions (i.e., adjusting line lengths at different orientations and at different positions within the figure).

As the positioning of the plastic sheets with the standard line took some time, there was always at least $10 \mathrm{sec}$ between trials. Ss were told they could request longer rest periods at any time they wished. After each trial, the length of the variable line was recorded to the nearest millimeter.

\section{RESULTS}

Illusion magnitude was determined by subtracting the length of the standard $(100 \mathrm{~mm})$ from the final setting of the variable (horizontal) line. The mean and stand ard error for each condition was calculated and 

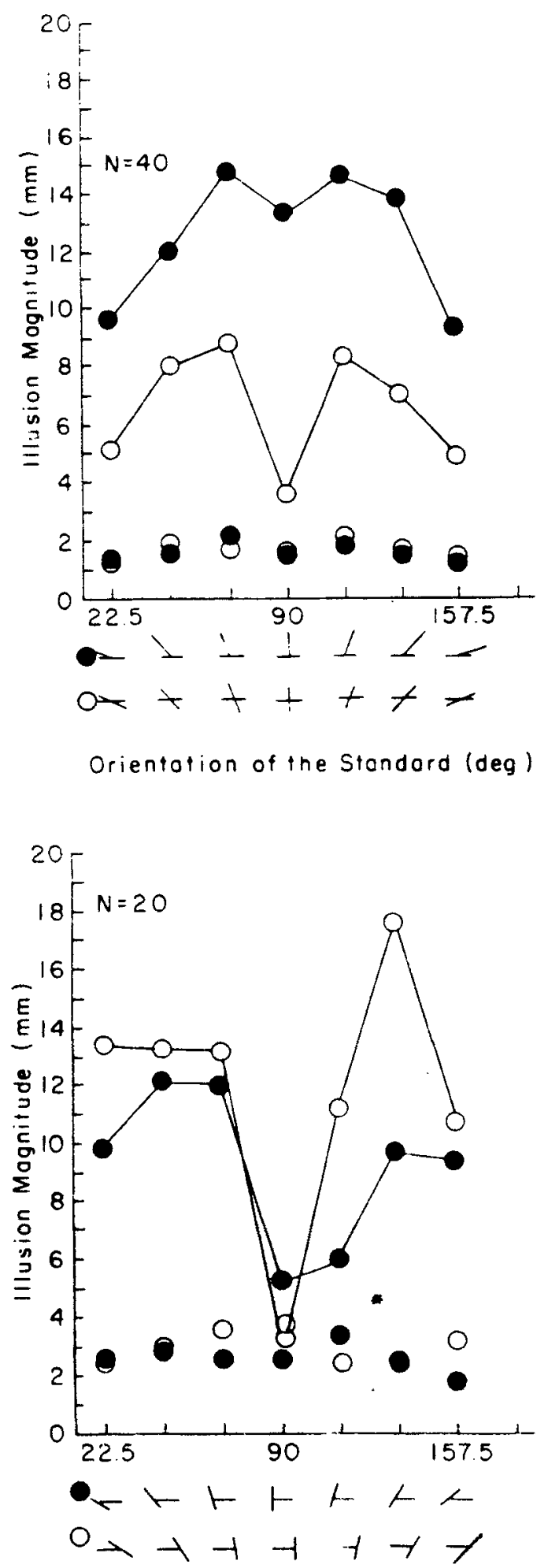

Orientation of the Standard (deg)

Fig. 1. Mean HVI magnitudes (connected circles) and standard errors (unconnected circles) as a function of stimulus configuration and inclination of the upright standard.

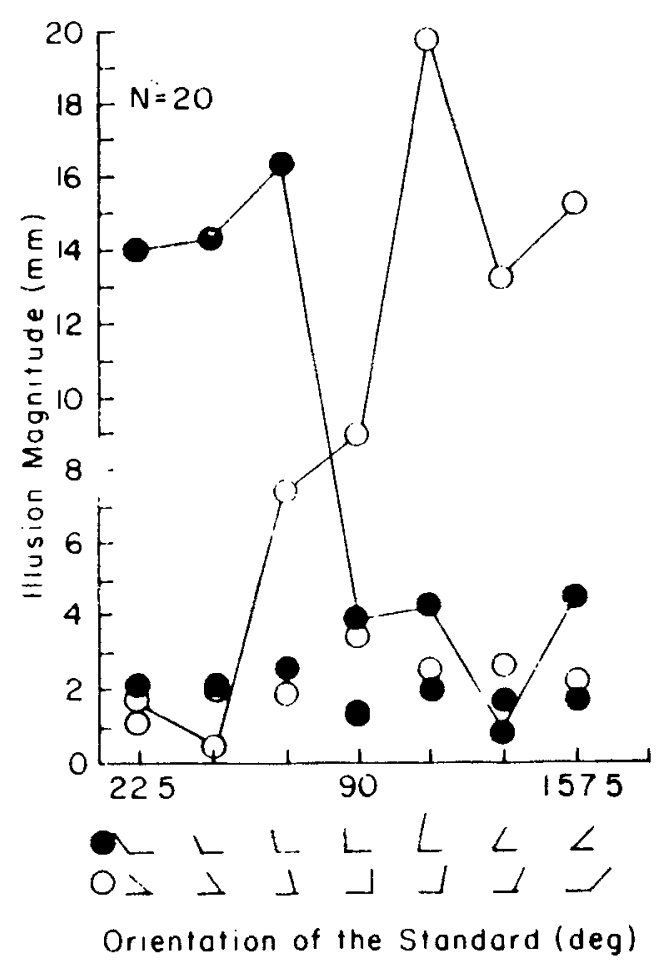

the results are shown in Fig. 1. The appropriate stimulus configuration for each data point is shown below the abscissa. The connected circles represent the mean illusion magnitudes in millimeters and the unconnected circles give the standard errors for the associated means.

The data for the two symmetrical figures (inverted $T$ and cross) were subjected to separate treatments (left vs right) by treatments (degree of tilt) by Ss ANOVAS. Degree of tilt was significant for both the cross $[F(2,78)=3.23, p<.05]$ and the inverted $T$ $[F(2,78)=5.1, p<.01]$. Neither direction of tilt nor interaction of direction vs degree of tilt were significant for either figure.

\section{DISCUSSION}

Inspection of Fig. 1 reveals that in no case did the true vertical standard $(90 \mathrm{deg})$ give the largest illusion; indeed, for some conditions it gave the smallest! Further, small deviations from the vertical in general yielded greater illusion magnitudes than did larger deviations. Finally, the largest illusion magnitudes and the smallest were produced by obtuse and acute angles, respectively, in conjunction with the $\mathrm{L}$ and reversed- $\mathrm{L}$ configurations.

Clearly, the effects of the orientation of the upright in the HVI are dependent upon the overall stimulus configuration. In the $L$ and reversed- $L$ figures, tilts which produced obtuse angles with the horizontal amplified the illusion, while acute angles attenuated 
it. We do not know what would happen if the angle between the two lines exceeded $157.5 \mathrm{deg}$ (the largest angle used). We might expect that the illusory effect would decline to zero as the angle approached $180 \mathrm{deg}$. Of course, it is possible that it would not.

The effects of acute angles on judgments involving the L figure in the present study parallel quite closely those found by Morinaga, Noguchi, and Ohishi (1962). They used an L figure with a small gap at the apex where the two lines would otherwise meet and empioyed angles of $90,75,60,45$, and $30 \mathrm{deg}$. They found a slight increase in the illusion as the upright inclined from 90 to $75 \mathrm{deg}$ and a rather steady decline thereafter. Several Ss in the present study commented that with the $L$ figures judgments were easiest with acute angles because the tips of the lines were close together and lengths could be equated by fairly simple alignment. A similar situation obtained with the cross when the upright was inclined to extremes in either direction.

The effects of tilt with the side $T$ and reversed side $T$ do not appear as clear-cut as those with the $L$ figures. The $90-\mathrm{deg}$ standard gave consistently the smallest illusory effect, and whether the standard was on the right or the left, the largest illusion was obtained when the horizontal formed an obtuse angle with the upper half of the upright. On the other hand, it is with these figures that we see the greatest differences between mirror image stimuli.

The relative potencies of the bisection illusion and the HVI are somewhat in doubt. Künnapas (1955a) and Tedford and Tudor (1969) found a negative HVI with the side-T figure, while Finger and Spelt (1947) and the present study found a small, but positive, HVI with this figure. Several differences in experimental design and stimulus conditions are correlated with the difference. Künnapas and Tedford and Tudor used a method of constant stimuli. The other two studies used a method of adjustment. In the present study and in that by Finger and Spelt, the standard line exceeded $3 \mathrm{deg}$ va in length and viewing distance exceeded $150 \mathrm{~cm}$. In the other two studies, the standard line was less than 3 deg va and viewing distance was $100 \mathrm{~cm}$ or less. There is evidence that HVI magnitude is inversely related to line length (Begelman \& Steinfeld, 1967), but no similar data are available for the bisection effect. Perhaps the most likely candidate as the variable producing a greater HVI in the present study is the so-called "error of the standard" (Gardner \& Long, 1960a, b) referred to in the method section above. When the vertical line is used as the standard with a method of adjustment, HVI measures are larger than when the horizontal line is used as the standard. In both the present study and that by Finger and Spelt, the standard line was vertical. The other studies did not employ the method of adjustment. In any case, the various dissimilarities among the studies comparing the HVI and bisection effect leave the differences in results not unreasonable.

The data from the cross and inverted $T$ are similar to those of Pollock and Chapanis (1952) and Shipley, Nann, and Penfield (1949), except for the lack of a right-left asymmetry as found by the former investigators. The symmetrical results of the present study also seem at variance with Underwood (1966).

The overall stimulus situations in these studies differ in many ways. Pollock and Chapanis employed two separate lines, while in the present study the lines formed a figure. The standard lines used by Pollock and Chapanis were either .96 or $1.91 \mathrm{deg}$ va in length and $.16 \mathrm{deg}$ va in width compared to $3.6 \mathrm{deg}$ va in length $x .07 \mathrm{deg}$ va in width for the present study. Pollock and Chapanis presented their black lines on a $22.6 \times 22.6$ deg va white square, while in the present study white lines were presented against a plain black background which filled the visual field. [Recall, however, that the, edges of the reduction screen aperture $(8.8 \times 7.1 \mathrm{deg}$ va) were faintly visible]. It is not intuitively apparent why any of these factors (or, for that matter, others not noted here) should lead to the obtained differences in results. It is not inconceivable that any of them might mask or overwhelm the tendency for lines tilted to the left to appest longer than those tilted to the right, but it is not immediately apparent why they might do so. It would seem equally reasonable to argue that no general tendency toward right-left asymmetry exists and that conditions specific to the previous studies produced the effect.

The general similarities noted between the results of the present study and those of Morinaga, Noguchi, and Ohishi (1962), Pollock and Chapanis (1952), and Shipley, Nann, and Penfield (1949) suggest that the failure to eliminate entirely the faint outline of the reduction screen was not severely detrimental. The similarities included results from asymmetric (L) and symmetric (inverted $T$ and cross) figures. The possible involvement of this factor in our failure to find right-left differences with symmetric figures has already been noted.

It is clear from Fig. 1 that the inverted $T$ yields larger illusion magnitudes than the cross, although otherwise their graphs are strikingly similar. The greater illusion magnitudes for the inverted-T figure probably represent the combined effects of the HVI and the bisection illusion as suggested by Künnapas (1955a) and Finger and Spelt (1947). In the cross, both lines are bisected, thus perhaps cancelling or attenuating the bisection effect.

It has already been noted that, in general, slight inclinations (22.5 deg from the vertical) of the upright lead to an increase in illusory effect. The only exception to this occurs with the reversed-L figure when the inclined line forms an acute angle with the horizontal. Greater inclinations often yielded large 
illusory effects, especially with the cross and side-T figures. These effects of tilt may reflect a basic anisotropy of the visual field. Pearce and Matin (1969) and Pearce and Taylor (1962) have found that judgments of linear extent (defined by points of light) vary as a function of orientation and retinal locus. Pearce and Matin suggest that these effects may be due to astigmatic properties of the refracting surfaces of the eye. While their data may not be directly relevant to a free-viewing situation, as in the present study, they are suggestive of a general tendency for judgments of length to be dependent on the orientation within the visual field.

Another possible explanation for these amplified effects with tilted lines derives from the perspective or inappropriate size-distance scaling hypothesis of visual illusions. According to this hypothesis, many visual illusions result from the perception of line drawings as three-dimensional figures (Gregory, 1966; Thiery, 1896; Woodworth \& Schlosberg, 1954). In the HVI, the vertical line may be seen to represent a foreshortened line extending into the distance (i.e., tilted away from the observer). The horizontal line, on the other hand, is seen as representing a line normal to the line of sight. If the images of the two lines subtend equal angles, then the vertical line must represent a greater extent, and perceptual constancy or size-distance mechanisms will make it appear longer.

Now, it might be argued that only rarely do lines or edges extend radially from the observer along his line of sight. Typically, they extend parallel to the line of sight (e.g., edges of roads, tables, and floors, as well as railroad tracks, lane markers, and the like), and thus in projection converge toward or away from it. Certainly in representations, contours extending into the distance are more often signified by lines tilted to the right or left of vertical than by vertical lines. Vertical objects, on the other hand, yield projections which are vertical and which do not represent foreshortened lines. Thus, if one accepts for the moment the perspective theory of visual illusions, one might further speculate that tilted lines are more likely to be seen in depth and therefore serve as more efficient triggers of the size-distance scaling mechanism and yield greater illusory effects. It must be noted that the perspective theory is not without difficulties (Over, 1968) and may not be able to account fully for all the visual illusions to which it has been applied. It is not unlikely, however, that perspective effects are involved in some visual illusions and may be important in explaining the effects of tilted uprights in the HVI.

In summary, the absence of asymmetric effects with our symmetrical figures (cross and inverted $T$ ) and the dramatic asymmetric effects with the other figures ( $\mathrm{L}$ and side $\mathrm{T}$ ) implicate specific stimulus conditions as the source of the right-left differences in tilt effects reported by previous authors. As regards the general effects of inclination of the upright, we advance cautiously the following interpretation. First, that inappropriate size-distance scaling is involved in the HVI. Second, that inclinations of the upright generate greater perspective effects and consequently lead to greater HVI magnitudes. Finally, when the stimulus configuration is such that tilting the upright brings the ends of the two lines into close juxtaposition (as with acute angles in the $L$ figures and the cross), comparisons of line length are simplified and the HVI is correspondingly reduced.

\section{REFERENCES}

Begelman, D. A., \& Steinfeld, G. An investigation of several parameters of the horizontal-vertical illusion. Perception \& Psychophysics, 1967, 2, 539-543.

Cormack, E. O. Effects of tilt of the upright in the horizontalvertical illusion. Unpublished paper, New Mexico Tech, 1969.

Cormack, E. O., \& Cormack, R. H. Interaction between stimulus configuration and line orientation in the horizontalvertical illusion. Paper presented at the meeting of the Rocky Mountain Psychological Association, Denver, May 1971.

Finger, F. W., \& SPELT, D. K. The illustration of the horizontalvertical illusion. Joumal of Experimental Psychology, 1947, 37. 243-250.

Gardner, R. W., \& Long, R. I. Errors of the standard and illusion effects with the inverted-T. Perceptual \& Motor Skills, $1960 \mathrm{a}, 10,47-54$.

GaRDNER, R. W., \& Long, R. 1. Errors of the standard and illusion effects with L-shaped figures. Perceptual \& Motor Skills, $1960 \mathrm{~b}, 10,107-109$.

Gregory, R. L. Eye and brain. New York: McGraw-Hill, 1966. Pp. $131-163$.

Künnapas, T. M. An analysis of the "vertical-horizontal illusion." Journal of Experimental Psychology, 1955a, 49, 134-140.

KüNNAPAs, T. M. Influence of frame size on apparent length of a line. Journal of Experimental Psychology, 1955b, 50, 168-170.

KünNaPAs, T. M. The vertical horizontal illusion in artificial visual fields. Journal of Psychology, 1959, 47, 41-48.

Morinaga, D., Noguchi, K., \& Ohishi, A. The horizontalvertical illusion and the relation of spatial and retinal orientations. Japanese Psychological Research, 1962, 4. 25-29.

Over, R. Explanations of geometrical illusions. Psychological Bulletin, 1968, 70, 545-562.

Pearce, D., \& Matin, L. Variations of the magnitude of the horizontal-vertical illusion with retinal eccentricity. Perception \& Psychophysics, 1969, 6, 241-243.

Pearce, D. G., \& Taylor, M. M. Visual length as a function of orientation at four retinal positions. Perceptual \& Motor Skills. $1962,14,431-438$.

Pollock, W. T., \& Chapanis, A. The apparent length of a line as a function of its inclination. Quarterly Journal of Experimental Psychology, 1952, 4, 170-178.

Shipley, W. C., Nann, B. M., \& Pennfield, M. J. The apparent length of tilted lines. Journal of Experimental Psychology. 1949, 39, 548-551.

TEDFord, W. H., JR., \& TuDor, L. L. Tactual and visual ilkasions in the $\mathrm{T}$-shaped figure. Journal of Experimental Psychology, 1969, 81, 199-201.

THIERY, A. Ueber geometrisch-optische Tauschungen. Phil. Stud., 1896, 12, 67-125. Cited by Borisc, E. G. Sensation and perception in the history of experimental psychology. New York: Appleton-Century-Crofts. 1942. P. 245.

UNDERWOOD, B. J. Experimental psychology. (2nd ed.) New York: Appleton-Century-Crofts, 1966. Pp. 68-98.

Woodworth, R. S., \& Schlosberg, H. Experimental psychology. (rev.) New York: Holt, 1954. P. 418.

(Received for publication September 7, 1973; revision received April 3, 1974.) 